\title{
Review
}

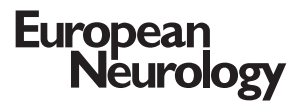

\section{Update on the Management of Hypertension for Secondary Stroke Prevention}

\author{
Luis Castilla-Guerra ${ }^{a}$ María del Carmen Fernández-Moreno ${ }^{\mathrm{b}}$ \\ ${ }^{a}$ Department of Internal Medicine, Hospital de la Merced, Osuna, and ${ }^{b}$ Department of Neurology, \\ Hospital de Valme, Sevilla, Spain
}

\section{Key Words}

Stroke $\cdot$ Risk factors $\cdot$ Hypertension $\cdot$ Blood pressure treatment $\cdot$ Stroke prevention $\cdot$ AHA/ASA guidelines

\begin{abstract}
High blood pressure (BP) is the strongest risk factor for stroke. It is estimated that almost $50 \%$ of strokes may be attributable to hypertension. Both diastolic and isolated systolic hypertension are important predictors of primary or recurrent strokes, and even minor decreases in BP can reduce the risk of stroke. While the primary prevention of stroke through the treatment of hypertension is well established, the issue of lowering BP after a stroke has been uncertain, particularly since this might worsen cerebral perfusion if autoregulation remains chronically damaged or severe carotid artery stenosis is present. Furthermore, there is substantial evidence to support BP lowering for prevention of a first stroke; however, few trials have focused on antihypertensive therapy for recurrent stroke prevention. In fact, currently, BP management in patients with strokes remains problematic, and questions such as the choice of antihypertensive drug and by how much to reduce BP are yet to be resolved. Recently, the American Heart Association and American Stroke Association published updated guidelines for recurrent stroke pre-
\end{abstract}

vention, and new recommendations on BP management have been included. Our review presents the most recent evidence on the management of hypertension in patients who have had a stroke.

Copyright $\odot 2012$ S. Karger AG, Basel

\section{Introduction}

High blood pressure (BP) is the strongest risk factor for stroke. It is estimated that almost $50 \%$ of strokes may be attributable to hypertension. Both diastolic and isolated systolic hypertension are important predictors of primary or recurrent stroke, and even minor decreases in $\mathrm{BP}$ can reduce the risk of stroke [1].

Whereas the primary prevention of stroke through the treatment of hypertension is well established, the issue of lowering BP after a cerebrovascular event has been uncertain, particularly since this might worsen cerebral perfusion if autoregulation remains chronically damaged or severe carotid artery stenosis is present $[2,3]$.

Furthermore, only few trials have focused on antihypertensive therapy for recurrent stroke prevention [3]. In fact, it was not until 2001, with the appearance of the results of the PROGRESS trial (Perindopril Protection

\section{KARGER \\ Fax +4161306 1234 \\ E-Mail karger@karger.ch}

www.karger.com
(C) 2012 S. Karger AG, Basel

0014-3022/12/0681-0001\$38.00/0

Accessible online at:

www.karger.com/ene
Dr. Luis Castilla Guerra

Calle de Juan Ramón Jiménez, 5

ES-41908 Castilleja de Guzmán (Spain)

E-Mail castillafernandez@ hotmail.com 
Against Recurrent Stroke Study), which was the first large-scale prospective trial published on the treatment of hypertension in the secondary prevention of stroke, when a clinical trial clearly demonstrated that antihypertensive treatment, this time with an angiotensin-converting enzyme inhibitor (ACEI), reduces the recurrence rate of stroke by $28 \%$ compared with placebo [4].

In a systematic review of 7 randomized controlled trials and 15,527 patients with a history of stroke, with and without hypertension, it was demonstrated that antihypertensive therapy reduces the risk of new stroke by $24 \%$ (OR 0.76, 95\% CI 0.63-0.92), myocardial infarction by $21 \%$ (OR 0.79, 95\% CI 0.63-0.98), and vascular events by $21 \%$ (OR $0.79,95 \%$ CI $0.66-0.95$ ), although not significantly reducing overall mortality or mortality due to stroke [3].

\section{The Evidence: Randomized Controlled Trials in Secondary Prevention of Stroke}

To date, only a few studies have been carried out on secondary prevention of stroke. Evidence from the main hypertension treatment trials on secondary stroke prevention is summarized in table 1 .

\section{PATS Trial}

The PATS (Post-Stroke Antihypertensive Treatment Study) trial [5] was a randomized, double-blind and placebo-controlled trial which aimed at determining whether antihypertensive treatment could reduce the risk of fatal and nonfatal stroke incidence in patients with a history of stroke or transient ischemic attack (TIA). In 5,665 patients, monotherapy with the diuretic indapamide (at doses of $2.5 \mathrm{mg} /$ day) was compared with placebo. The findings of this trial indicated that in patients with a history of stroke or TIA, BP reduction of $5 / 2 \mathrm{~mm} \mathrm{Hg}$ with indapamide reduced the incidence of fatal and nonfatal stroke by $29 \%$.

\section{PROGRESS Trial}

The PROGRESS trial [4] randomized 6,105 patients with a history of ischemic stroke or TIA with and without hypertension to 3 groups that received perindopril $(4 \mathrm{mg}$ / day) alone, in combination with indapamide $(2.5 \mathrm{mg} /$ day), or placebo.

After a mean follow-up of 3.9 years, the active treatment group as a whole had significantly fewer strokes and major vascular events. On average, treatment reduced systolic $\mathrm{BP}$ (SBP) by $9 \mathrm{~mm} \mathrm{Hg}$ and diastolic $\mathrm{BP}$ (DBP) by
$4 \mathrm{~mm} \mathrm{Hg}$, and reduced the relative risk of a stroke by $28 \%$ (95\% CI 17-38) versus placebo. However, a prespecified subgroup analysis showed that although participants treated with the combination of perindopril plus indapamide had an average decrease of $12 \mathrm{~mm} \mathrm{Hg}$ in SBP and $5 \mathrm{~mm} \mathrm{Hg}$ in DBP and had a significantly lower stroke risk than patients who received double placebo ( $43 \%$ risk reduction, 95\% CI 30-45\%), patients treated with perindopril alone showed a $\mathrm{BP}$ reduction of $5 / 3 \mathrm{~mm} \mathrm{Hg}$ and had a stroke risk that was not discernibly different from placebo (5\% risk reduction, $95 \% \mathrm{CI}-19$ to $23 \%$ ).

\section{MOSES Trial}

The MOSES trial [6] was the first to compare the relative benefit of particular different antihypertensive drugs in the secondary prevention of stroke. It compared the efficacy of treatment with eprosartan (an angiotensin II type receptor blocker; $600 \mathrm{mg} /$ day) and nitrendipine (a calcium channel blocker) in 1,045 hypertensive patients who had a cerebral event in the previous 24 months. BP reductions were similar with the two agents. Treatment with eprosartan resulted in a $25 \%$ stroke risk reduction when compared to nitrendipine (236 events; incidence density ratio $0.75,95 \%$ CI $0.58-0.97$ ), and there was also a reduction in the risk of primary composite events (death, cardiovascular event, or cerebrovascular event; incidence density ratio $0.79,95 \%$ CI $0.66-0.96$ ). A reduction in TIAs accounted for most of the benefit in cerebrovascular events, with no significant difference in ischemic strokes, and a more traditional analysis of time to first cerebrovascular event did not show a benefit of eprosartan. However, the study had an open design, amongst other methodological limitations.

\section{PRoFESS Trial}

In the PRoFESS (Prevention Regimen for Effectively Avoiding Second Strokes) trial [7], 20,332 patients with recent ischemic stroke (within 90 days of the event) were randomized to either the angiotensin receptor blocker (ARB), $80 \mathrm{mg}$ /day telmisartan $(\mathrm{n}=10,146)$, or placebo $(\mathrm{n}=10,186)$ on a background of standard antihypertensive therapy as part of a $2 \times 2$ factorial design study, which also included aspirin plus extended-release dipyridamole versus clopidogrel. Telmisartan was not associated with a reduction in recurrent stroke (HR 0.95, 95\% CI 0.86-1.04) or major cardiovascular events (HR 0.94, 95\% CI 0.87-1.01) during a mean follow-up of 2.5 years. The BP-lowering arm in PRoFESS was statistically underpowered. Nonadherence to telmisartan and more aggressive treatment with other antihypertensive medica- 
Table 1. Trials on blood pressure lowering and secondary stroke prevention

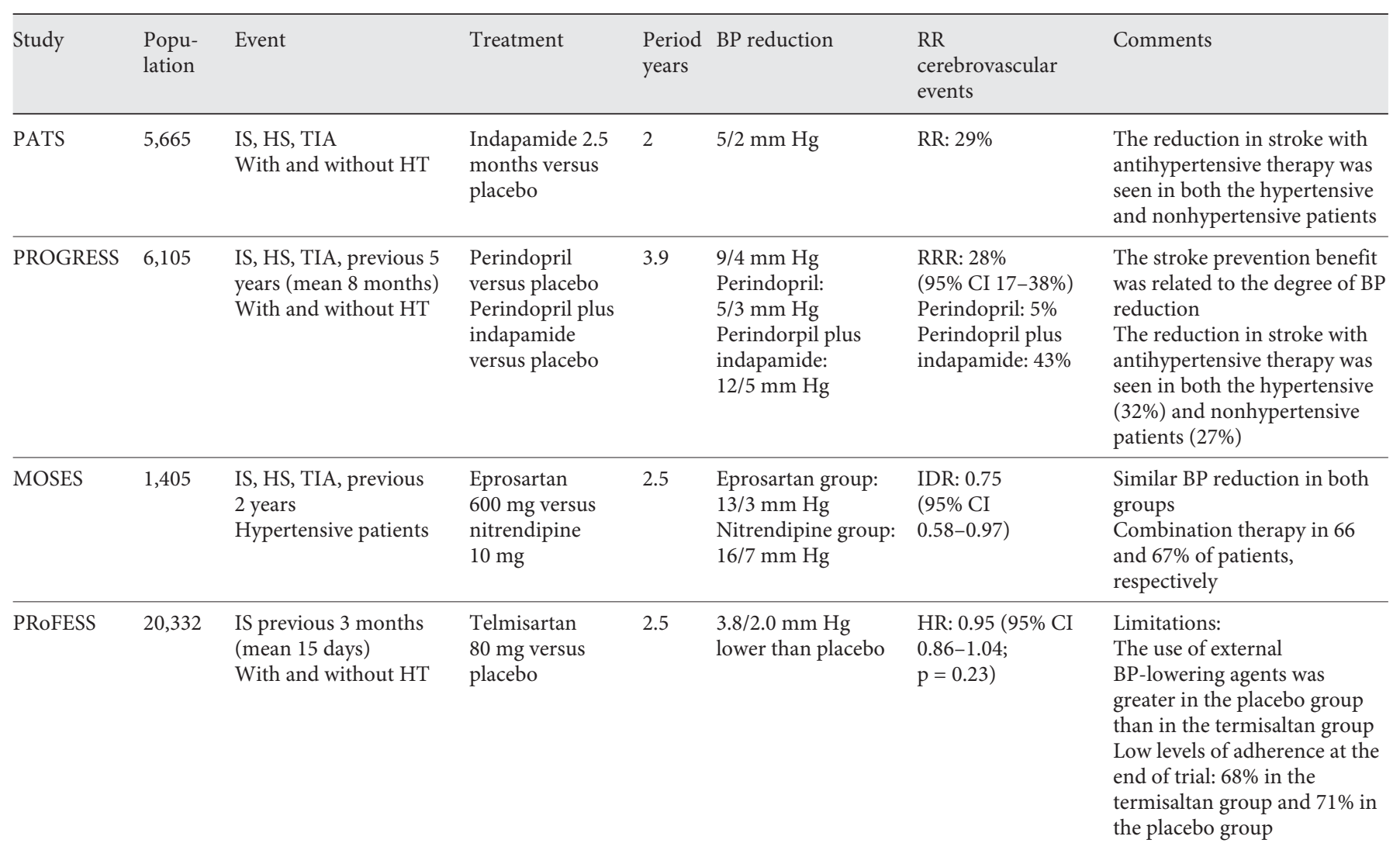

IS = Ischemic stroke; HS = hemorrhagic stroke; RR = risk reduction; RRR = relative risk reduction; IDR = incidence density ratio; $\mathrm{HT}=$ hypertension.

tions in the placebo group reduced the difference in $\mathrm{BP}$ between the treatment groups (SBP differed by $5.4 \mathrm{~mm}$ $\mathrm{Hg}$ at 1 month and $4.0 \mathrm{~mm} \mathrm{Hg}$ at 1 year) and may have reduced the impact of treatment on stroke recurrence [3].

\section{Is It Safe to Lower BP in Patients Who Have Had a Stroke for the Purpose of Recurrent Stroke Prevention?}

Although there is now unanimity on the benefit of lowering BP in patients with stroke, there is no consensus over the target $\mathrm{BP}$ levels in secondary stroke prevention $[3,8]$, with some controversy still remaining over whether long-term antihypertensive treatment may compromise cerebral perfusion in patients with prior stroke, especially in elderly subjects with carotid disease $[2,3]$.

Thus, excessive reduction in BP, especially at night either naturally or through the use of antihypertensive drugs, could lead to unwanted ischemic events, such as subcortical silent cerebral infarction (associated with Binswanger's disease) or ocular symptoms (e.g., anterior ischemic optic neuropathy) [9]. This could be especially important in subjects who have suffered a stroke, in which cerebral autoregulation, which is responsible for maintaining cerebral blood flow constant over a wide range of $\mathrm{BP}$, is often dysfunctional, causing cerebral perfusion becoming more dependent on the values of $\mathrm{BP}$ [10].

Cerebral resistance vessels in normotensive individuals are known to maintain cerebral blood flow relatively constant between 50 and $150 \mathrm{~mm}$ Hg mean arterial pressure. Long-standing hypertension in itself shifts the lower limits of the autoregulatory threshold upward; even after effective and long-standing antihypertensive treatment, readaptation of cerebral autoregulation is not always completed, so that the lower limits of autoregulatory thresholds are still higher than in normotensive subjects. Thus, it is possible that important decreases in BP could result in more severe decreases in cerebral blood flow if pressure 
falls below the lower limit of autoregulation [10]. In addition, the chronic elevation of $\mathrm{BP}$ would be associated with nonreversible hypertensive vascular structural changes, and hence functional vascular readaptation is not likely to take place when BP is lowered $[9,10]$.

The normal response to uncontrolled hypertension is arteriolar thickening in the cerebral and other vascular districts; this is in part an appropriate adaptation in that it prevents the increased pressure from being transmitted to the capillary circulation. Although it protects the capillary circulation, arteriolar thickening also limits the ability to maintain perfusion as hypertension is treated [11]. A possible marker for underlying cerebral arteriolar disease is the finding of copper wiring or silver wiring in the retinal arterioles on fundoscopy examination. These changes reflect arteriolar thickening as progressively less and eventually, with silver wiring, none of the blood streams can be visualized through the arteriolar wall [11].

Moreover, in hypertensive patients also coronary autoregulation in the subendocardium is shifted toward higher pressure, probably owing to left ventricular hypertrophy and functional and structural alterations in the small coronary vessels, causing an impaired tolerance to BP decrease [10].

All these alterations would facilitate the presence of silent cerebrovascular and cardiac ischemic damage in these same patients and could possibly increase vascular morbidity and mortality and the risk for recurrent strokes.

Another group of patients more sensitive to these episodes of hypotension would include those with hemodynamically significant carotid occlusive disease, in which $\mathrm{BP}$ falls distal to the stenosis when luminal narrowing in the carotid artery is $\geq 70 \%$ or when the residual lumen diameter is $\leq 2 \mathrm{~mm}$. In such cases, and in the absence of adequate collateral circulation, a low cerebral perfusion pressure can cause ischemia in the border areas between vessels, especially in cases where the collateral circulation to the brain is altered by an incomplete circle of Willis, or occlusion of the contralateral carotid artery or the basilar artery $[2,12]$.

One study addressed this issue by looking at the relationship between BP and risk of stroke in medically treated patients in two major randomized trials of carotid endarterectomy and one major randomized trial of aspirin [13]. The risk of stroke increased with increasing $\mathrm{BP}$ in patients with symptomatic carotid artery disease and, to a similar degree, in patients with unilateral carotid artery occlusion. In contrast, among patients with bilateral carotid stenosis of $\geq 70 \%$, the risk was inverted, and patients with lower BP had more events.

Recently, it has been shown that patients with previous ischemic stroke in the middle cerebral artery have an impaired cerebral perfusion. In these patients, the velocities of basal cerebral blood flow were $30 \%$ lower than in subjects without prior stroke, and cerebral flow velocities were more dependent on BP values [14].

Therefore, gradual BP reduction is indicated in patients with known cerebrovascular disease, especially with long-standing uncontrolled hypertension.

\section{What Is the Target BP Goal in Patients Who Have Had a Stroke?}

Another important issue is the target $\mathrm{BP}$ in these patients. In daily clinical practice in secondary stroke prevention it is recommended to keep BP below $130 \mathrm{~mm} \mathrm{Hg}$ SBP and $85 \mathrm{~mm} \mathrm{Hg} \mathrm{DBP,} \mathrm{preferably} \mathrm{with} \mathrm{an} \mathrm{ACEI} \mathrm{and/or}$ diuretic (level I evidence, grade A recommendation) [3]. However, these BP figures may be too strict for subjects with recent or subacute stroke and, possibly, may too often cause hypotension at night, with the resulting additional risk to these patients. In fact, over the last years, there has been a return to the classic debate on the J-curve in BP.

In line with what some authors have argued for decades and based on the results of several studies such as INVEST (International Verapamil Trandolapril Study) [15] and ONTARGET (Ongoing Telmisartan Alone and in Combination with Ramipril Global Endpoint Trial) [16], the concept of 'the lower the better' is under review and should probably be abandoned. In fact, although national and international guidelines recommend aggressive $\mathrm{BP}$ control to $<140 / 90 \mathrm{~mm} \mathrm{Hg}$ for uncomplicated hypertension or $<130 / 80 \mathrm{~mm} \mathrm{Hg}$ for hypertension associated with coronary artery disease, diabetes, or chronic kidney disease, recent large clinical outcome trials have observed a J-curve effect between a DBP of $<80 \mathrm{~mm} \mathrm{Hg}$ as well as a SBP of $<130 \mathrm{~mm} \mathrm{Hg}$ and have cast some doubt regarding aggressive $\mathrm{BP}$ treatment. As these investigators noted, these recommendations were informed by wisdom, not facts, and in most clinical trials which have produced benefits from the treatment of hypertension, BP was seldom reduced to $140 / 90 \mathrm{~mm} \mathrm{Hg}[17,18]$.

This is especially important in the elderly. In a recent meta-analysis on the treatment of BP in subjects aged 80 years or older, the greatest reduction in mortality occurred in subjects with less intensive therapy [19]. 
Nevertheless, in contrast to most clinical trials demonstrating a definite J-curve effect between low DBP and cardiovascular complications, there are several trials in which the J-curve effect was not clearly evident for stroke. The most likely explanation is that, in contrast to coronary circulation which depends on DBP, the cerebral circulation depends mostly on SBP. Therefore, these studies would show that cerebral blood flow is not seriously affected even with very low DBPs, and this could explain the lack of a J-curve effect regarding strokes with low DBPs in contrast to coronary artery disease complications [18].

On the other hand, major guideline recommendations to lower BP in patients with a history of cerebrovascular disease even when its values are in the high-normal or normal range could be based on a not entirely appropriate interpretation of the results of the PROGRESS trial [4], the study that unequivocally showed the benefits of lowering $\mathrm{BP}$ in patients with a previous stroke. The original publication reported significant reductions in stroke and major cardiovascular events both in hypertensive and nonhypertensive patients, but hypertension was defined by a SBP of at least $160 \mathrm{~mm} \mathrm{Hg}$ or a DBP of at least $90 \mathrm{~mm} \mathrm{Hg}$, irrespective of treatment (50\% of the patients were under antihypertensive treatment). Indeed, in a subsequent publication in which baseline SBP values were stratified in greater detail, significant benefits appeared to occur in patients with a baseline SBP of at least $160 \mathrm{~mm} \mathrm{Hg}$ or between 140 and $159 \mathrm{~mm} \mathrm{Hg}$, but not in those at lower baseline SBP values, in which the significant difference with the placebo group was lost [20]. This subanalysis also provided no evidence of a J-curve relationship between BP level and stroke risk among patients with cerebrovascular disease.

Besides, in a recent review on the aims of antihypertensive therapy in patients with prior stroke, the target of $\mathrm{SBP}<130 \mathrm{~mm} \mathrm{Hg}$ was not obtained in any of the trials: $143 \mathrm{~mm} \mathrm{Hg}$ in the PATS, $141 \mathrm{~mm} \mathrm{Hg}$ in the PROGRESS, $150 \mathrm{~mm} \mathrm{Hg}$ in the ACCESS (Acute Stroke Therapy in Cilexetil Candesartan Survivors) [21], and $136 \mathrm{~mm} \mathrm{Hg}$ in the PRoFESS [17]. Therefore, these questions are still to be answered in patients with established cerebrovascular disease.

\section{Which BP-Lowering Agent Is Most Effective in Patients Who Have Had a Stroke?}

In the last few years, evidence from experimental and clinical studies has supported the hypothesis that angiotensin II (Ang II) might exert detrimental effects beyond
Table 2. AHA/ASA recommendations for BP management for prevention of recurrent stroke

1. BP reduction is recommended for both prevention of recurrent stroke and prevention of other vascular events in persons who have had an ischemic stroke or TIA and are beyond the first $24 \mathrm{~h}$ (class I; level of evidence A).

2. Because this benefit extends to persons with and without a documented history of hypertension, this recommendation is reasonable for all patients with ischemic stroke or TIA who are considered appropriate for BP reduction (class IIa; level of evidence B).

3. An absolute target BP level and reduction are uncertain and should be individualized, but benefit has been associated with an average reduction of approximately $10 / 5 \mathrm{~mm} \mathrm{Hg}$, and normal BP levels have been defined as $<120 / 80 \mathrm{~mm} \mathrm{Hg}$ by JNC 7 (class IIa; level of evidence B).

4. Several lifestyle modifications have been associated with BP reduction and are a reasonable part of a comprehensive antihypertensive therapy (class IIa; level of evidence C).

These modifications include salt restriction; weight loss; consumption of a diet rich in fruits, vegetables, and low-fat dairy products; regular aerobic physical activity; and limited alcohol consumption.

5. The optimal drug regimen to achieve the recommended level of reduction is uncertain because direct comparisons between regimens are limited. The available data indicate that diuretics or the combination of diuretics and an ACEI are useful (class I; level of evidence A).

6. The choice of specific drugs and targets should be individualized on the basis of pharmacological properties, mechanism of action, and consideration of specific patient characteristics for which specific agents are probably indicated (e.g., extracranial cerebrovascular occlusive disease, renal impairment, cardiac disease, and diabetes) (class IIa; level of evidence B) (new recommendation).

the mechanical damage of high BP and might be a risk factor for ischemic stroke independent of its effect on BP. Thus, data from the HOPE (Heart Outcomes Prevention Evaluation) [22] and LIFE (Losartan Intervention For Endpoint reduction in hypertension) [23] studies suggested that ACEIs and ARBs may have protective effects for stroke that are independent of BP reduction [24].

Activation of the renin-angiotensin-aldosterone (RAS) system plays an important role in the pathophysiology of hypertension and atherosclerotic vascular disease. Besides, ARBs attenuate RAS system activation by competitively inhibiting the binding of Ang II to AT1 receptors while allowing for unopposed stimulation of the AT2 receptors. AT1 blockade interrupts a negative feed- 
back loop leading to a 3- to 5-fold increase in Ang II levels from baseline with hyperstimulation of AT2 receptors. AT2 activation is hypothesized to protect against stroke by recruiting cerebral collateral vessels and enhancing neuronal resistance to anoxia and by attenuating prothrombosis, inflammation, and endothelial dysfunction, which mediate atherosclerosis [25].

However, in view of recent published trials, data on the particular benefits of these specific antihypertensive agents for secondary stroke prevention are largely lacking. First, the PROGRESS trial provided no evidence of perindopril alone for preventing recurrent stroke. In fact, only the subgroup receiving both perindopril in combination with indapamide had reduced stroke recurrence [4]. Furthermore, there is no evidence to date that using perindopril in combination with indapamide is more beneficial than using indapamide alone. Thus, the BP reduction with indapamide alone in the PATS trial was only $5 / 2 \mathrm{~mm} \mathrm{Hg}$, similar to the reduction seen with perindopril alone in the PROGRESS, and, on the contrary, it was significantly associated with stroke reduction $[4,5]$.

Results from the ARB segment of the PRoFESS trial were unable to show any significant reduction in the risk of a subsequent stroke or of the composite outcome of major cardiovascular events with telmisartan use [8]. The authors have pointed out several study limitations that may have led to the observed results: the mean duration of 2.5 years of follow-up may have been too short to demonstrate any significant benefit with telmisartan; there was low adherence to telmisartan (by 36 months, adherence to telmisartan had dropped to $68 \%$ ), and the DBP $(<80 \mathrm{~mm} \mathrm{Hg})$ in the first months after stroke may have been too low in at least one third of the population with a baseline SBP $<130 \mathrm{~mm} \mathrm{Hg}$, because a high dose of telmisartan was given after a very short post-stroke delay (mean 15 days) without discontinuation of baseline antihypertensive treatment [26].

However, authors have also suggested a time-dependent effect of telmisartan. Thus, in a post-hoc analysis, from 6 months, telmisartan significantly reduced the number of strokes versus placebo (533 vs. 608 ; HR 0.88 , 95\% CI 0.78-0.99; $\mathrm{p}=0.042$ ) [27]. Recently, the results of a meta-analysis of 20 randomized clinical trials with 108,286 patients with stroke revealed that ARBs provide benefit on preventing stroke when compared with placebo (OR 0.91, 95\% CI 0.84-0.98). However, there was no evidence of the benefit when comparing ARBs with ACEIs (OR 0.93, 95\% CI 0.84-1.03) and with calcium antagonists (OR 1.16, 95\% CI 0.91-1.48) [28]. Therefore, in general, all major classes of BP-lowering agents may di- minish recurrent stroke risk, and the degree of BP reduction may be more important than the class of the agent used.

\section{Guidelines}

Recently, a joint committee representing the American Heart Association (AHA) and the American Stroke Association (ASA) has published updated evidence-based recommendations on the prevention of ischemic stroke among survivors of ischemic stroke or TIA [3]. Table 2 lists recommendations for $\mathrm{BP}$ management according to AHA/ASA guidelines. In these new recommendations, authors remark that the choice of specific antihypertensive drugs and $\mathrm{BP}$ targets should be individualized on the basis of pharmacological properties, mechanism of action, and consideration of specific patient characteristics for which specific agents are probably indicated (e.g., extracranial cerebrovascular occlusive disease, renal impairment, cardiac disease, and diabetes).

\section{Conclusions}

Hypertension is not only the major risk factor for primary stroke, but it also increases the risk of recurrent strokes and cardiovascular morbidity and mortality in patients who have had a stroke. In view of recent published trials, currently, there is evidence supporting the use of BP-lowering agents in patients who have had a stroke. However, BP management in these patients remains problematic, and questions such as the choice of antihypertensive drug and by how much to reduce $\mathrm{BP}$ are matters for debate.

Recent AHA/ASA guidelines recommend that the choice of specific antihypertensive drugs and BP targets should be individualized on the basis of pharmacological properties, mechanism of action, and consideration of specific patient characteristics for which specific agents are probably indicated. Nevertheless, further studies will be required to clearly determine the optimal management of $\mathrm{BP}$ and the use of antihypertensives in this patient population. 


\section{References}

$>1$ Lawes CM, Bennett DA, Feigin VL, Rodgers A: Blood pressure and stroke: an overview of published reviews. Stroke 2004;35:776-785.

$>2$ Castilla-Guerra L, Fernández-Moreno M del C, Alvarez-Suero J: Secondary stroke prevention in the elderly: new evidence in hypertension and hyperlipidemia. Eur J Intern Med 2009;20:586-590.

-3 Furie KL, Kasner SE, Adams RJ, et al: Guidelines for prevention of stroke in patients with ischemic stroke or transient ischemic attack: a statement for healthcare professionals from the American Heart Association/American Stroke Association Council on Stroke: cosponsored by the Council on Cardiovascular Radiology and Intervention: the American Academy of Neurology affirms the value of this guideline. Stroke 2011;42:227-276.

$\checkmark 4$ PROGRESS Collaborative Study Subgroup: Randomized trial of perindopril based blood pressure-lowering regimen among 6,108 individuals with previous stroke or transient ischemic attack. Lancet 2001;358:1033-1041.

$>5$ PATS Collaborating Group: Post-stroke antihypertensive treatment study: a preliminary result. Chin Med J (Engl) 1995;108:710 717.

6 Schrader J, Luders S, Kulschewski A, et al: Morbidity and mortality after stroke - eprosartan compared with nitrendipine for secondary prevention: principal results of a prospective randomized controlled study (MOSES). Stroke 2005;36:1218-1226.

7 Yusuf S, Diener HC, Sacco RL, et al: Telmisartan to prevent recurrent stroke and cardiovascular events. N Engl J Med 2008;359: 1225-1227.

$>8$ Castilla-Guerra L, Fernández-Moreno $\mathrm{M}$ del C, Espino-Montoro A, López-Chozas JM Ambulatory blood pressure monitoring in stroke survivors: do we really control our patients? Eur J Intern Med 2009;20:760-763.

$\checkmark 9$ Sare GM, Gray LJ, Wardlaw J, Chen C, Bath PM, ENOS Trial Investigators: Is lowering blood pressure hazardous in patients with significant ipsilateral carotid stenosis and acute ischaemic stroke? Interim assessment in the 'Efficacy of Nitric Oxide in Stroke' trial. Blood Press Monit 2009;14:20-25.
10 Ruland S, Aiyagari V: Cerebral autoregulation and blood pressure lowering. Hypertension 2007;49:977-978.

11 Kaplan NM, Rose BD: Treatment of hypertension in patients who have had a stroke. www.uptodate.com (accessed September, 2010)

12 Kantola I, Terent A, Kataja M, Breig-Asberg E: Ace-inhibitor therapy with spirapril increases nocturnal hypotensive episodes in elderly hypertensive patients. J Hypertens 2001; 15:873-878.

13 Rothwell PM, Howard SC, Spence JD, the Carotid Endarterectomy Trialists' Collaboration: Relationship between blood pressure and stroke risk in patients with symptomatic carotid occlusive disease. Stroke 2003;34: 2583-2590.

14 Novak V, Hu K, Desrochers L, et al: Cerebral flow velocities during daily activities depend on blood pressure in patients with chronic ischemic infarctions. Stroke 2010;41:61-66.

15 Pepine CJ, Handberg EM, Cooper-DeHoff $\mathrm{RM}$, et al: A calcium antagonist vs. a noncalcium antagonist hypertension treatment strategy for patients with coronary artery disease. The International Verapamil-Trandolapril Study (INVEST): a randomized controlled trial. JAMA 2003;290:2805-2816.

16 The ONTARGET Investigators: Telmisartan, ramipril, or both in patients at high risk for vascular events. N Engl J Med 2008;358: 1547-1559.

17 Zanchetti A, Grassi G, Mancia G: When should antihypertensive drug treatment be initiated and to what levels should systolic blood pressure be lowered? A critical reappraisal. J Hypertens 2009;27:923-934.

18 Chrysant SG: Effectiveness of lowering blood pressure to prevent stroke versus to prevent coronary events. Am J Cardiol 2010; $106: 825-829$.
19 Bejan-Angoulvant T, Saadatian-Elahi M, Wright JM, Schron EB, Lindholm LH: Treatment of hypertension in patients 80 years and older: the lower the better? A meta-analysis of randomized controlled trials. J Hypertens 2010;28:1366-1372.

20 Arima H, Chalmers J, Woodward M, et al, the PROGRESS Collaborative Group: Lower target blood pressures are safe and effective for the prevention of recurrent stroke: the PROGRESS trial. J Hypertens 2006;24:12011208.

21 Schrader J, Lüders S, Kulschewski A, et al: The ACCESS Study: evaluation of Acute Candesartan Cilexetil Therapy in Stroke Survivors. Stroke 2003;34:1699-1703.

-22 Yusuf S, Sleight P, Pogue J, Bosch J, Davies R, Dagenais G: Effects of an angiotensin-converting-enzyme inhibitor, ramipril, on cardiovascular events in high-risk patients. The Heart Outcomes Prevention Evaluation Study Investigators. N Engl J Med 2000;342: 145-153.

23 Lindholm LH, Ibsen H, Dahlöf B, et al: Cardiovascular morbidity and mortality in patients with diabetes in the Losartan Intervention For Endpoint reduction in hypertension study (LIFE): a randomised trial against atenolol. Lancet 2002;359:1004-1010.

24 Castilla-Guerra L, Fernández-Moreno MC, Jiménez-Hernandez MD: Blockage of the renin-angiotensin system in the secondary prevention of stroke: beneficial effects beyond blood pressure reduction? Stroke 2009; 40:e75.

25 Strauss MH, Hall A: Angiotensin receptor blockers should be regarded as first-line drugs for stroke prevention in both primary and secondary prevention settings: no. Stroke 2009;40:3161-3162.

26 Diener HC, Wong P: Development in secondary stroke prevention. Eur Neurol Rev 2008;48:50-57.

-27 Diener HC: Preventing stroke: the PRoFESS, ONTARGET, and TRANSCEND trial programs. J Hypertens Suppl 2009;27:S31-S36.

-28 Lu GC, ChengJW, Zhu KM, et al: A systematic review of angiotensin receptor blockers in preventing stroke. Stroke 2009;40:38763878 . 